\title{
Die französischen Gewerkschaften in der Wirtschaftskrise: Zwischen Dialogue Social und Basismilitanz
}

Während in Deutschland die befürchteten sozialen Unruhen während der Wirtschaftskrise ausblieben, kam es in Frankreich im Frühjahr und Sommer $2009 \mathrm{zu}$ Massendemonstrationen und diversen spektakulären „Bossnapping“-Aktionen. Wie lassen sich die deutlich unterschiedlichen Konfliktkulturen in Deutschland und Frankreich erklären? Welche gesetzlichen Regeln, institutionellen Rahmenbedingungen sowie historischen Entwicklungen beeinflussen die nationalen Systeme industrieller Beziehungen? Und welche Missverständnisse sind typisch, wenn aus deutscher Sicht die französischen Verhältnisse betrachtet werden?

\section{Einleitung}

Wenn deutsche Gewerkschafterinnen und Gewerkschafter eine interessenbewusstere Politik einklagen, sprechen sie nicht selten davon, man „müsse endlich ein bisschen französisch lernen“. Dieses Stereotyp französischer Gewerkschaftsmilitanz erhielt vor Kurzem neue Nahrung, als im Zuge der Wirtschaftskrise in spektakulärer Weise Reifenhaufen brannten und medienträchtige „Bossnapping“-Aktionen stattfanden. Aber stimmt es wirklich, dass in unserem westlichen Nachbarland die Gewerkschaften einen so viel kämpferischeren Politikstil pflegen? Und wenn ja, mit welchem Erfolg? Wie sahen die gewerkschaftlichen Strategien während der Wirtschaftskrise aus? Unterschieden sie sich wirklich so deutlich von den deutschen Verhältnissen?

Um diese Fragen zu beantworten, werden im Folgenden zunächst vergleichend einige Charakteristika der industriellen Beziehungen in Deutschland und Frankreich rekapituliert. Anschließend wird dargestellt, welche Strategien die französischen Gewerkschaften im Krisenverlauf ergriffen haben und in welchem Kontext z. B. die spektakulären „Bossnapping“-Aktionen zu verorten sind. ${ }^{1}$ Im abschließenden Resümee wird die These vertreten, dass die gewerkschaftlichen Krisenreaktionen sich diesseits und jenseits des Rheins im Grunde stark ähneln: Die Gewerkschaften bewähren sich in erster Linie als Ordnungsmacht. Sie setzten auf die Wahrung der Interessen ihrer Stammklientel durch den sozialen Dialog mit der Regierung. Während diese
Politik allerdings in Deutschland weitgehend bruchlos an sozialpartnerschaftliche Traditionslinien anschließt, bedeutet sie in Frankreich eine graduelle Neujustierung etablierter Politikmuster.

\section{Industrielle Beziehungen in Deutschland und "à la francaise $^{\prime \prime}$}

Deutschland und Frankreich werden häufig im internationalen Maßstab als ähnliche Gesellschafts- und Wirtschaftsmodelle diskutiert, etwa als „koordinierte Marktwirtschaften" (Hall/Soskice 2001) oder „rheinische Kapitalismen“ (Albert 1991; Streeck 1999). Identisches Merkmal beider Länder ist eine vergleichsweise flächendeckende sowie einheitliche Regulierung der Arbeitsbedingungen. Der zentrale Unterschied liegt darin, dass diese in Deutschland vor allem durch die Tarifverbände erfolgt, in Frankreich in erster Linie vom Staat geleistet wird. Dieser ist nicht nur selbst der größte Arbeitgeber, er setzt mit dem Mindestlohn SMIC (salaire minimum interprofessionnel de croissance) auch die Löhne von ca. 13 \% der Beschäftigten direkt fest (z.Zt. in Höhe von $8,86 €$ brutto pro Stunde). Zugleich sichert er die Gültigkeit von Tarifverträgen, indem er sie häufig für allgemeinverbindlich erklärt. Deutschland gilt daher in der Literatur vielfach als Modellbeispiel sozialpartnerschaftlicher Konzertation oder sogar neokorporatistischer Arrangements, Frankreich hingegen als etatistisch reguliertes System (vgl. Berger 1981; Traxler et al. 2001; Hassel 2009; Rehfeldt 2009).
Der jeweilige Ort der Gewerkschaften im System nationaler politischer Ökonomie korrespondiert mit unterschiedlichen nationalen Organisationstraditionen sowie differenten rechtlichen Regelungen der Konfliktaustragung zwischen Arbeit und Kapital. Das deutsche System gilt als „verrechtlichtes“ System der Interessenvertretung (Erd 1978), in dem die Mobilisierung von Primärmacht legal nur in eingeschränktem Maße möglich ist und repräsentatives Stellvertreterhandeln von Gewerkschaften und Betriebsräten eine große Rolle spielt. Die Voraussetzungen, um unter diesen Bedingungen eine Kultur der „Konfliktpartnerschaft“ (MüllerJentsch 1993) zu etablieren, waren traditionell ein hoher Zentralisierungs- sowie Organisationsgrad der Tarifverbände sowie solide rechtliche Machtressourcen der Betriebsräte. Das intermediär angelegte, sozialpartnerschaftliche Regulierungssystem in Deutschland wird häufig als idealtypisch für koordinierte Marktwirtschaften (Hall/Soskice 2001) sowie vorbildhaft für den allgemeinen Trend in Europa gesehen.

\footnotetext{
Grundlage der folgenden Darstellung sind mehrere Interviews mit wissenschaftlichen und gewerkschaftlichen Experten und Expertinnen, die Anfang Februar 2010 in Paris geführt wurden. Ein besonderer Dank für viele wertvolle Hinweise gilt Jean-Marie Pernot, Institut de Recherches Economiques et Sociales (IRES).
}

\footnotetext{
Ingrid Artus, Prof. Dr., Friedrich-AlexanderUniversität Erlangen, Institut für Soziologie. Arbeitsschwerpunkte: Vergleichende Gesellschaftsanalyse, Industrielle Beziehungen, Prekäre Arbeitsverhältnisse. e-mail: ingrid.artus@soziol.phil.uni-erlangen.de
} 
Frankreich firmiert hingegen als „Spezialfall“ (Amadieu 1995), als ,atypisch “ und „anormal“ (Goetschy 1998). Deviant sind die französischen industriellen Beziehungen insbesondere wegen ihrer anhaltend hohen Konfliktintensität bei zugleich ausgesprochen schwacher gewerkschaftlicher Organisierung. Hintergrund ist ein ausgeprägter Gewerkschaftspluralismus, der sich in den letzten 20 Jahren noch verstärkt hat. Eine anhaltend klassenkämpferische Rhetorik (eines dominanten Teils) der Gewerkschaften korrespondiert mit vitalen Traditionen rigider patronaler Herrschaft. Die schwachen rechtlichen Ressourcen der betrieblichen Vertretungsinstitutionen bei zugleich starkem individuellen Streikrecht favorisieren eine ausgesprochen dezentrale Gewerkschaftskultur. Arbeitskämpfe sind oft eine notwendige Vorbedingung von Verhandlungen und zur Etablierung eines rapport de force (Machtverhältnisses) im Betrieb (vgl. Artus 2008). Die politische Tätigkeit der Gewerkschaftszentralen ist eher an den Staat als an die Unternehmer und ihre Verbände adressiert. Die ausgeprägte Demonstrations- und Protestkultur der französischen Nation, deren Gründungsmythologie sich auf eine bürgerliche Revolution bezieht, sorgt zudem für eine hohe Akzeptanz vergleichsweise militanter Aktionen, die eher als produktives Element der Zivilgesellschaft interpretiert werden denn als nachhaltige Gefährdung der öffentlichen Ordnung. Zu Beginn der 1990er Jahre war angesichts des massiven Mitgliederverlusts der Gewerkschaften zuweilen über ein Ende der „Ausnahmeerscheinung “französischer Konfliktkultur spekuliert worden. Die massenhaften Streiks und Demonstrationen der Jahre 1995, 2003 und zuletzt 2006 gegen den Contrat Première Embauche (Ersteinstellungsvertrag) bewiesen jedoch nachdrücklich das Gegenteil. Die Zahl der offiziell registrierten ausgefallenen Arbeitstage im Zeitraum 1995 bis 2006 je 1.000 Beschäftigte lag mit 91,4 Tagen im Jahresdurchschnitt etwa 25 mal so hoch wie der deutsche Wert (3,6 Tage) (vgl. Dribbusch 2008).

\section{Gewerkschaftliche Strukturkrise - westlich wie östlich des Rheins}

Der wirtschaftliche und gesellschaftliche Strukturwandel seit den 1970er Jah- ren führte in Frankreich etwas früher, in Deutschland etwas später zu einer deutlichen Verschiebung der Machtverhältnisse zuungunsten der abhängig Beschäftigten (Artus 2010). In beiden Ländern sind seit den 1990er Jahren Tendenzen zur Dezentralisierung, Fragmentierung und Verbetrieblichung der Verhandlungen zwischen Kapital und Arbeit festzustellen (Pernot 2005; Artus 2008). Es muss von einer Krise der Gewerkschaften gesprochen werden, die sich sowohl in einer Organisationskrise (nach innen) als auch in der Erosion gewerkschaftlicher Einflussmacht (nach außen) zeigt:

Die Mitgliederkrise der Gewerkschaften ist in Frankreich wesentlich gravierender als in Deutschland. Zwischen 1979 und 1988 halbierte sich der gewerkschaftliche Organisationsgrad und nahm auch in der Folgezeit kontinuierlich ab bis auf heute nur noch etwa 7 bis $8 \%$. Frankreich hat damit den niedrigsten gewerkschaftlichen Organisationsgrad aller OECD-Länder. Von den noch etwa 1,8 Mio. französischen Gewerkschaftsmitgliedern sind $13 \%$ verrentet (Andolfatto/Labbé 2009, S. 15f.) und ein Großteil ist im öffentlichen Dienst tätig. Die Privatwirtschaft kann, von einigen Inseln (in oft ehemals staatlichen) Großbetrieben der Automobil-, Luftfahrt-, Chemie- und Mineralölindustrie sowie einigen Banken und Versicherungen abgesehen, als nahezu gewerkschaftsfreier Bereich gelten. Die Gewerkschaften stehen zunehmend im Verdacht, von den Verhältnissen in Privatunternehmen nur noch eine vage Ahnung zu besitzen.

Auch die Fragmentierung der Gewerkschaftslandschaft ist in Frankreich wesentlich massiver als in Deutschland - und sie nimmt weiter zu. Die Zahl der relevanten gewerkschaftlichen Organisationen hat sich von ehemals fünf staatlicherseits als repräsentativ anerkannten Gewerkschaftsverbänden (CGT, CFDT, CFTC, CFE-CGC, FO) inzwischen auf acht erhöht (inclusive FSU, Solidaires, UNSA) ${ }^{2}$.

Identisch sind in beiden Ländern allerdings die eklatanten Probleme bei der Organisierung jener „neuen“ Arbeitnehmerinnen und Arbeitnehmer, deren Anteil immer mehr zunimmt: Frauen, jüngere Menschen, Beschäftigte des Dienstleistungssektors sowie prekär Beschäftigte.

Die Erosion gewerkschaftlicher Einflussmacht dokumentiert sich in Frankreich in einem Prozess des toujours moins (immer weniger) (Andolfatto/Labbé 2009).
Obwohl die französische Wirtschaftspolitik auf eine Förderung der Binnennachfrage orientiert ist (Horn et al. 2008) und die Steigerung der Reallöhne im vergangenen Jahrzehnt (2000-2009) mit 6,7\% über dem deutschen sowie dem EU-weiten Durchschnitt lag (Schulten 2010), war dieser Zuwachs doch sehr ungleich verteilt. Laut Andolfatto/Labbé (2009, S. 7) wuchs zwischen 1998 und 2006 die Kaufkraft der französischen Haushalte zwar um ca. 5 \%, die ärmeren $50 \%$ derselben profitierten davon jedoch nicht. Hinzu kommt, dass die konservative französische Regierung die Arbeitszeitverkürzung der 1990er Jahre mittlerweile durch diverse Gesetzesnovellen aufgeweicht hat. Arbeitsintensität, Leistungsdruck und Beschäftigungsprekarität nehmen zu. Seit Anfang der 1980er Jahre hat sich die Zahl der Praktikums- und staatlich unterstützten Beschäftigungsverhältnisse (contrats aidés) verdreifacht, die der befristeten Verträge vervierfacht und die Zeitarbeit hat sich verfünffacht (IRES 2009, S. 40f.). 2008 lag die Arbeitslosenquote mit ca. $8 \%$ etwa gleichauf mit der BRD, jedoch deutlich über dem Wert der OECD (5,7 \%) (ebd., S. 38). Die viel diskutierten Selbstmordwellen in französischen Großunternehmen wie Renault und Telecom sind sicherlich der dramatischste Ausdruck des zunehmenden Leidens (auch) am französischen Arbeitsplatz (vgl. Dejours/ Bègue 2009).

Auch in Frankreich hat die größte globale Wirtschaftskrise seit 1929 also aus Sicht der Gewerkschaften und vieler Beschäftigter als „Krise in der Krise“ zu gelten. Die Desyndikalisierung und der Verlust gewerkschaftlicher Einflussmacht sind „das Prisma“, durch das hindurch die Gewerkschaftskader die Wirtschaftskrise wahrnahmen und das den Interpretationsrahmen stellte (Béroud/Yon 2009). Die Strategie, die in dieser Situation ergriffen wurde, ist eine, die angesichts unsicherer und krisenhafter Umweltfaktoren Handlungssicherheit und -stabilität erhöhen sollte. So waren die gewerkschaftlichen Krisenpolitiken von einem ungewöhn-

\footnotetext{
2 CGT = Confédération générale du travail; CFDT = Confédération française démocratique du travail; CFTC $=$ Confédération française des travailleurs chrétiens; CFE-CGC = Confédération française de l'encadrement - Confédération générale des cadres; FO = Force Ouvrière; FSU = Fédération Syndicale Unitaire; Solidaires $=$ Union Syndicale Solidaires; UNSA = Union Nationale des Syndicats Autonomes.
} 
lichen Willen zur Einheit geprägt - und zugleich von einem ausgeprägten Attentismus sowie „relativer Ängstlichkeit“ der Gewerkschaftszentralen in der Wahl ihrer Aktionsmittel (ebd.). Angesichts der eigenen Schwäche zögerten sie, den dialogue social mit der Regierung aufzukündigen, da das Einschlagen eines Konfrontationskurses als eine zu risikoreiche und in ihren Auswirkungen schwer überschaubare Strategie eingeschätzt wurde. Sie zögerten so lange, bis sich die Möglichkeit eines solchen Kurses von selbst erledigt hatte.

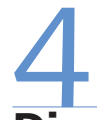

Die Ausgangssituation: La loi sur la représentativité

Auch ohne Einsetzen der Wirtschaftskrise wäre 2008 für die französischen Gewerkschaften ein einschneidendes Jahr gewesen: Am 20.August 2008 wurde nach langer Diskussion das Gesetz über die Neuordnung der Regeln zur Repräsentativität verabschiedet (la loi sur la représentativé). Es reformiert das System industrieller Beziehungen in Frankreich in grundlegender Weise. Nach Ende des Zweiten Weltkriegs waren fünf Gewerkschaften staatlicherseits pauschal als repräsentativ anerkannt worden - ein Status, der sie zu diversen wichtigen staatlichen Organisationshilfen berechtigt. So errangen etwa ihre Vertreter in den Betrieben (délégués syndicaux) 1968 das Recht, betriebliche Verträge abzuschließen - unabhängig vom jeweiligen betrieblichen Organisationsgrad (vgl. Artus 2008). Das neue Gesetz sieht nun vor, dass die Gewerkschaften ihre Repräsentativität auf Betriebs- sowie Branchenebene fortan nicht mehr pauschal staatlicherseits verliehen bekommen, sondern über eine Mindestverankerung nachzuweisen haben. In klassisch liberaldemokratischer Weise soll dies auf dem Weg von Wahlen geschehen. Vertragsfähig sind im Betrieb zukünftig nur noch Gewerkschaften, die während der Vertreterwahlen (für das comité d'entreprise bzw. die délégués du personnel) mehr als $10 \%$ der Wählerstimmen erhalten (8\% auf Branchenebene). ${ }^{3}$ Die neue Regelung, über deren prinzipielle Notwendigkeit sich weite Kreise von politischen, wissenschaftlichen sowie gewerkschaftlichen Experten einig sind, erschüttert derzeit die französische Betriebs- und Gewerkschaftslandschaft und ist in ihren
Auswirkungen noch kaum abschätzbar. Vermutlich wird sie insbesondere die großen Gewerkschaften (CGT und CFDT) stärken, die das Gesetz daher auch explizit mittrugen. Zugleich ist absehbar, dass aufbetrieblicher Ebene vielfach die Karten neu gemischt werden. Die jeweils kleineren Gewerkschaften gehen Bündnisse ein, um weiterhin verhandlungsfähig zu bleiben. Angesichts der buntscheckigen französischen Gewerkschaftslandkarte bedeutet dies jedoch, dass an differenten Orten jeweils unterschiedliche Gewerkschaften miteinander koalieren (müssen) - was die Heterogenität der betrieblichen Verhältnisse möglicherweise noch steigern könnte. Die Regelung konstituiert jedenfalls einen neuen Zwang zur Zusammenarbeit sowie zur verstärkten Basisorientierung. Das Gesetz stellt die tradierten Verhandlungsroutinen vielerorts infrage, und es kommt zu Prozessen der Rejustierung und Neuausbalancierung betrieblicher Machtverhältnisse, die die französische Gewerkschaftslandschaft wohl für diverse Jahre prägen werden.

Kurz nach Einsetzen der Wirtschaftskrise, am 3. Dezember 2008, fanden in Frankreich die ersten Beschäftigtenwahlen nach Verabschiedung der loi sur la représentativitéstatt. Es handelte sich zwar nicht um die betrieblichen Vertreterwahlen, sondern um jene für die prud'hommes (LaienArbeitsgerichte), angesichts der neuen Bedeutung betrieblicher Wahlprozesse waren diese dennoch eine wichtige Generalprobe. ${ }^{4}$ Somit waren die gewerkschaftlichen Kader massiv mit Wahlkampf beschäftigt, als der französische Präsident Sarkozy im Herbst 2008 den ersten nationalen Aktionsplan zur Krisenbekämpfung ins Leben rief. Erst am 5. Januar 2009 reagierten die Gewerkschaften politisch auf die sich zuspitzende Wirtschaftskrise, indem sie eine "gemeinsame Plattform“ deklarierten.

\section{Mobilisierung von oben: Gewerkschaftseinheitlicher Attentismus und Priorität des Dialogue Social}

Die „Forderungsplattform für den Streik am 29. Januar 2009“" war von allen acht größeren Gewerkschaften unterschrieben und ausgesprochen allgemein gehalten. Das Plattformpapier betont, dass der Markt nicht alle Probleme regeln könne, fordert Vorrang für eine Politik der Beschäftigungsstabilisierung, eine Bekämpfung der Wirtschaftskrise durch Steigerung der Binnennachfrage, die Reduktion von Einkommensungleichheiten, die Aufrechterhaltung sozialer Errungenschaften und kollektiver Absicherungen sowie die Regulierung des internationalen Finanzmarktes. In der öffentlichen Diskussion wurde das Papier als grobe Skizze eines ökonomischen und sozialen Gegenprogramms zur Regierungspolitik aufgefasst, deren zentraler Protagonist Sarkozy zu diesem Zeitpunkt ausgesprochen niedrige Popularitätswerte verzeichnete. Als solches wurde es auch von weiteren linken Organisationen und Parteien unterstützt.

Der gewerkschaftlich geeinte Aufruf zu landesweiten Demonstrationen und Streiks am 29. Januar 2009 fand ein riesiges Echo. Nach Gewerkschaftsangaben waren zwischen 2 und 2,5 Mio. Menschen auf den Straßen. Die Bewegung hatte damit „aus dem Stand" ein Ausmaß, das mit den Höhepunkten der Jahre 1995, 2003 und 2006 vergleichbar war. Die Demonstrationen waren zudem von einer beeindruckenden Streikbewegung in den Betrieben begleitet - ein Umstand, auf den sich die französischen Gewerkschaften zunehmend weniger verlassen können. Angesichts der Arbeitsmarktmisere und des gestiegenen Leistungsdrucks in den Betrieben geben viele Beschäftigte ihrem Unmut lieber auf der Straße Ausdruck als am Arbeitsplatz. Es gibt einen gewissen Trend dahin gehend, dass die zivilgesellschaftliche Demonstration den betrieblichen Streik ersetzt (Pernot 2010). Trotz der offenbar ungewöhnlich hohen Protestbereitschaft der Beschäftigten war der Aktionstag jedoch nicht wie 2006 während der erfolgreichen Bewegung gegen den contrat première embauche (CPE) der Auftakt zu wöchentlichen Mobilisierungen, die letztlich das Ausmaß einer Kraftprobe zwischen den Regierenden und „der Straße“ annahmen. Vielmehr entschieden die Gewerkschaften, erst sechs

3 Vgl. für genauere Angaben die nach Betriebsund Branchenebene differenzierten neuen Regelungen Ministère du Travail 2008.

4 Die CGT erhielt bei den Wahlen im Dezember $200833,8 \%$ der Stimmen und gewann damit 1,6 Prozentpunkte seit 2002 hinzu. Die CFDT erhielt $22,1 \%(-3 \%)$, FO $15,9 \%(-2,3 \%)$, die CFTC $8,9 \%(-0,7 \%)$, die CFE-CGC $8,2 \%(+1,2 \%)$, UNSA 6,6 \% (+1,2\%), Solidaires 3,8 \% (+2,3\%). Es beteiligten sich jedoch nur etwa $25 \%$ der stimmberechtigten Beschäftigten an den Wahlen. 
Wochen später zu einem neuen Aktionstag zu mobilisieren. Die Resonanz am 19. März 2009 war, gemessen an der Zahl der Demonstrierenden, sogar noch größer als im Januar. Manche Kommentatoren waren der Ansicht, dass es sich um die größten Demonstrationen seit 1968 gehandelt habe. Doch was tun mit dieser kollektiven Wut (colère collective)? Die Idee eines eintägigen oder gar unbefristeten Generalstreiks stand im Raum und wäre der naheliegende nächste Schritt gewesen, hätte man die Mobilisierung vorantreiben und auf eine neue Eskalationsstufe heben wollen.

Aber nichts dergleichen geschah. Die Gewerkschaften einigten sich vielmehr darauf, den traditionell arbeitsfreien 1. Mai zum nächsten Aktionstag und damit Streiks für hinfällig zu erklären. Der gemeinsame Aufruf aller Gewerkschaften zu Demonstrationen am 1. Mai war für Kenner der französischen Geschichte durchaus ein historischer Moment, da sich der explizit antikommunistische Gewerkschaftsverband FO bis dato nie an den gemeinsamen 1.-Mai-Demonstrationen beteiligt hatte. In Zeiten der Krise präsentierten sich die Gewerkschaften also symbolisch in nie dagewesener Einheit - einer Einheit, die im Zeichen der Mäßigung und Konfliktregulierung stand. Für die politische Mobilisierung hatte diese Entscheidung fatale Folgen. Zwar war die Beteiligung an den 1.-Mai-Demonstrationen deutlich stärker als in „normalen“ Jahren, es kamen aber weniger Demonstrantinnen und Demonstranten als noch im März. Die kollektive Wut hatte einen Dämpfer erfahren angesichts des augenfälligen Willens der Gewerkschaftsführungen, eine direkte Konfrontation mit der Regierung zu vermeiden. Als die Gewerkschaften dann am 7. Juni 2009 in vorbildlicher Einheit zu einem weiteren Aktionstag aufriefen, war dieser ein völliger Misserfolg. Wie ist dieser - auf den ersten Blick durchaus dysfunktionale und erstaunliche - Wille zur Mäßigung ausgerechnet von Seiten der konfliktgewohnten französischen Gewerkschaften zu erklären?

\section{Der gewerkschaftliche Wille zur Mäßigung: Erklärungsansätze}

Die oben skizzierte „Verschleppungstaktik“ ist zunächst sicherlich Ausdruck des
Primats zur Wahrung der Gewerkschaftseinheit. Diese war 2006 als wesentliches Element der erfolgreichen Bewegung gegen den Contrat première embauche erlebt worden - im Gegensatz zur gescheiterten Massenmobilisierung im Jahr 2003, bei der die CFDT aus der gewerkschaftlichen Einheitsfront ausgeschert war. Interpretiert durch das „Prisma“ gewerkschaftlicher Krise, hatte das Verhindern (erneuter) gewerkschaftlicher Spaltung deutliche Priorität. Im Rahmen einer stark pluralistischen Gewerkschaftslandschaft impliziert dies jedoch die Notwendigkeit, kompromisshaft vermittelte Minimalpositionen zu beziehen - eine sicherlich ungünstige Voraussetzung, um das Risiko eines Konflikts mit der Regierungsgewalt einzugehen. Die Entscheidung für einen Generalstreik hätte jedenfalls mit großer Wahrscheinlichkeit die Einheit im Gewerkschaftslager gesprengt. Obwohl der gewöhnlich eher an pragmatischer Tarifpolitik orientierte, traditionell antikommunistische Gewerkschaftsbund FO seine Vertreter explizit ermächtigte, für die Idee eines eintägigen Generalstreiks einzutreten, hätten sich andere politisch gemäßigte Gewerkschaftsbünde, wie etwa die CFDT und die CGC, kaum daran beteiligt.

Letztlich entscheidend war aber wohl die Haltung des größten Gewerkschaftsverbandes, der ehemals kommunistischen CGT. Sie hielt „den Schlüssel“ zum Generalstreik „in der Hand“ (Béroud/Yon 2009) - und entschied sich dafür, ihn nicht zu benutzen. „La grève générale ne se decrète pas“ (der Generalstreik lässt sich nicht verordnen) (Interviewzitat CGT), betonten hohe Funktionsträgerinnen und -träger und verlagerten damit die Entscheidungshoheit über die Ausweitung der Kampfmaßnahmen gleichsam auf den spontanen Willen der Basis, für den man nicht verantwortlich sei. Zwei Hintergründe sind maßgeblich, um dieses Verhalten zu verstehen:

Es ist zunächst Ausdruck der ideologischen Kehrtwendung, die die (ehemals stalinistisch geprägte) CGT etwa Mitte der 1990er Jahre vollzogen hat - unter dem Eindruck des Zusammenbruchs des realsozialistischen Blocks sowie massiv schwindender Mitgliederzahlen. Um als fille ainée älteste Tochter) der kommunistischen Partei Frankreichs nicht in vernichtender Weise in deren Abwärtsstrudel hineingezogen zu werden, löste man die ehemals strikte organisatorische Anbindung und
Unterordnung gewerkschaftlicher Belange unter die ideologischen Prämissen der Partei. Obwohl CGT-intern freilich viele Traditionsbestände der kommunistischen Arbeiterbewegung bis heute überlebt haben, ging mit dieser Umorientierung eine deutliche Entideologisierung einher. Statt umfassenderer politischer Forderungen wurden „rein“ gewerkschaftliche Zielsetzungen in den Vordergrund gestellt. Nicht mehr objektiv gegebene Interessen der Arbeiterklasse wollte man vertreten, sondern nunmehr die subjektiven Meinungen derselben in Erfahrung bringen. Statt als Vorhut und Kaderschmiede der Arbeiterklasse interpretierte man die eigene Rolle zunehmend bescheidener: Es gehe darum, „ein offenes Ohr" für die Probleme und Wünsche der Beschäftigten zu haben - „être à l'écoute“ (Zuhören).

Die ideologische Wende war erfolgreich. Gegen Ende der 1990er Jahre zeichnete sich immer deutlicher das ,unglaubliche Überleben der CGT“ (Mouriaux 1998) ab. Sie ist - nach dem Aderlass der CFDT im Gefolge ihres Ausscherens aus der Gewerkschaftseinheit im Jahr 2003 - wieder der größte französische Gewerkschaftsverband. Und doch ist ihre gewerkschaftliche Identität erschüttert, das Vertrauen in die eigene Stärke geschwunden. Man geht „den Massen“ nicht mehr voran, sondern läuft ihnen im Zweifelsfall hinterher, ängstlich bemüht, den Anschluss nicht zu verpassen. Die verschleppte Mobilisierung im Frühjahr 2009 ist somit auch als Ausdruck ihrer verinnerlichten Schwäche zu interpretieren.

Andererseits ist die mäßigende Haltung der CGT in Zeiten zugespitzter gesellschaftlicher Konflikte so neuartig auch wieder nicht - man denke etwa an die Mai-Unruhen von 1968 (vgl. GilcherHoltey 1995). Ohne hier nicht vorhandene historische Parallelen konstruieren zu wollen, zeigt sich doch in beiden Fällen die typische Rolle aller großen französischen Gewerkschaftsdachverbände (nicht nur der CGT): Während der wiederkehrenden sozialen Bewegungen in der französischen Geschichte reagieren sie eher auf die Ereignisse, versuchen die dezentralen Aktivitäten zu koordinieren und „einzufangen“; sie betätigen sich als Sprachrohre und Mediatoren, treiben die soziale Unruhe im Regelfall nicht noch voran, sondern bemühen sich darum, diese zu nutzen, indem sie sie in handfeste Ergebnisse und Verträge ummünzen. Dabei sind ihre 
Qualitäten als Ordnungsmacht historisch durchaus variabel. Nicht immer gelang es ihnen, durch ihre Verhandlungsergebnisse die Unruhe an der Basis zu befrieden. Gerade angesichts der volatilen Strukturen gesellschaftlicher Gegenmacht und der ausgeprägten Protestkultur werden die Gewerkschaften jedoch in ihrer Rolle als Mediatoren und Ordnungskräfte vom französischen Staat hoch geschätzt - und als solche auch umfassend finanziell gefördert. Die erheblichen staatlichen Organisationshilfen und finanziellen Unterstützungsleistungen spielen bekanntlich eine wesentliche Rolle für die organisatorische Funktionsfähigkeit der französischen $\mathrm{Ge}$ werkschaftszentralen (vgl. Jefferys 2003, S. 213f.). Seit der virulenten gewerkschaftlichen Mitgliederkrise gilt dies mehr denn je.

Bezogen auf die gewerkschaftliche Politik während der Wirtschaftskrise bedeutet dies, dass sich die Gewerkschaften von der Aufrechterhaltung des dialogue social vermutlich mehr versprachen als von dessen Aufkündigung. Dies gilt insbesondere für die CGT, die seit der Entideologisierung und Pragmatisierung ihrer Politik einen deutlich größeren politischen Spielraum für Verhandlungen mit der Regierung besitzt. Dass die loi sur la représentativité insbesondere die beiden großen Gewerkschaftsbünde (CGT und CFDT) stärkt, ist politisch aus Regierungssicht erst opportun, seit die CGT nicht mehr (zumindest ideologisch) als eine Art „innerer Feind“ zu gelten hat. Der Weg zu ihrer verstärkten Einbindung in das politische System Frankreichs ist nun frei und aus Sicht der Gewerkschaftsführungen wohl auch mit diversen Chancen verbunden. So hielt die CGT zwar „den Schlüssel“ zum Generalstreik ,,in der Hand“. Sie war aber offenbar nicht bereit, in turbulenten Zeiten sowie angesichts fraglicher eigener Stärke ihre Rolle als Dialogpartner der Regierung aufzugeben zugunsten einer riskanten Strategie der Basismobilisierung mit ungewissem Ausgang.

\section{Mobilisierung von unten: Betriebsbesetzungen und "Bossnapping"}

Schon im März und April 2009 hatten parallel zum Aufschwung der landesweiten Mobilisierung „von oben“ - einzelne besonders militante Aktionen gegen Entlassungen und für erhöhte Abfindungszahlungen ein großes Medienecho erregt, so etwa der Fall des Baumaschinenherstellers Caterpillar in Grenoble, wo der Generaldirektor, der Personalchef, der Qualitätschef und der Europachef des Unternehmens für 25 Stunden von „ihren“ Arbeitern in Arrest genommen wurden (vgl. Hahn 2009a). Ähnliche Aktionen hatte es kurz zuvor auch bei Sony und der Firma $3 \mathrm{M}$ (Pithiviers) gegeben. Im April machten die Arbeiterinnen und Arbeiter des von Schließung bedrohten Continental-Werks in Clairoix Schlagzeilen, als sie nicht nur Reifen verbrannten und die örtliche Präfektur zerlegten, sondern auch mit einem Sonderzug zur Unternehmenszentrale nach Hannover reisten, um dort zu demonstrieren (vgl. hierzu kritisch Wildcat 2009).

Im Mai und Juni 2009 verstärkte sich der Prozess forcierter Entlassungen in vielen französischen Unternehmen. Dieser wurde in wesentlich geringerem Maße als in Deutschland durch Kurzarbeit und Arbeitszeitflexibilisierung aufgefangen und gemildert. ${ }^{5}$ Die Kräfte vieler gewerkschaftlicher Aktivisten waren fortan im Rahmen betrieblicher Feuerwehrpolitik gebunden. Besonders betroffen waren kleinere Zulieferbetriebe, denen als „Puffer" die Aufträge gekündigt wurden. Die Großunternehmen konnten den Auftragsrückgang z.T. durch Insourcing und Erhöhung der Fertigungstiefe ausgleichen, um so ihr Stammpersonal weiter zu beschäftigen. Diese Praxis bedeutete jedoch das Aus für viele kleinere Zulieferbetriebe, vor allem der Automobilindustrie. Zeitlich gesehen fiel der Perspektivenverlust der colère collective daher mit einer forcierten colère éclatée zusammen - einer explodierenden Wut in der Form lokal zersprengter Einzelaktionen.

Medienberichten zurfolge wurden z. B. beim Klebstoffhersteller Scapa in Bellegarde-sur-Valserine, beim größten französischen Autozulieferer Faurecia (in der Nähe von Paris), beim Reifenhersteller Michelin (Werk Blanzy) und in der Batteriefabrik Fulmen (in Auxerre/Burgund) vorübergehend Managerinnen und Manager festgesetzt. Besonders bekannt wurde im Juli 2009 der Fall der 366 Beschäftigten des Autoteileherstellers New Fabris im westfranzösischen Châtellerault. Sie installierten auf ihrem Fabrikdach 19 Gasflaschen und drohten, diese zu sprengen, wenn ihre
Forderung nach erhöhten Abfindungszahlungen nicht erfüllt würde. Bezahlen sollten die Prämien die beiden langjährigen Hauptkunden der Fabrik, Renault und PSA. Auch beim Netzwerkausrüster Nortel erreichten die Beschäftigten nach der Androhung der Sprengung ihres von der Schließung bedrohten Werks deutlich erhöhte Abfindungszahlungen. Obwohl örtlich vielfach Gewerkschaftsaktivisten zu den Organisatoren zählten, blieben die gewerkschaftlichen Zentralen diesen Aktionen gegenüber meist indifferent oder betonten sogar, „Gewalt führe zu nichts“. Etwas verlegen machte sie wohl auch der Umstand, dass i.d.R. nicht der Erhalt von Arbeitsplätzen im Vordergrund stand, sondern erhöhte Abfindungszahlungen. Diese inhaltliche Bestimmung der Kämpfe muss sicherlich im Kontext der parachutes dorées (goldenen Fallschirme) gesehen werden, die zeitgleich für viele Manager aufgespannt wurden. Sie kann jedoch auch als Ausdruck einer Desillusionierung bezüglich der französischen staatlichen Beschäftigungspolitik (des reclassements) gelesen werden. Und es waren Aktionen, die angesichts des Scheiterns kollektiver Strategien von einem Gefühl des „rette sich wer kann“ geprägt waren (vgl. ähnlich Beaud/Pialoux 2004). Das konnten allerdings bei Weitem nicht alle.

\section{Deutsch-französische Missverständnisse}

Die Militanz der französischen Aktionen wurde und wird in Deutschland häufig als Ausdruck einer erheblichen Kampfstärke der französischen Arbeiterinnen und Arbeiter, wenn nicht sogar der Gewerkschaften gewertet. Dieser Eindruck ist jedoch in mehrfacher Hinsicht zu relativieren:

\footnotetext{
Dies liegt vor allem daran, dass Kurzarbeit (chômage partiel oder chômage technique) in Frankreich nur kurzfristig einsetzbar ist. Zudem ist sie auch gesetzlich weniger lukrativ gestaltet. Immerhin sind die Regelungen im Zuge der Wirtschaftskrise verbessert worden: Im Januar 2009 wurde das Kurzarbeitergeld auf $60 \%$ des ausgefallenen Arbeitsentgelts erhöht (zuvor $50 \%$ ) und ist nun für höchstens 800 (in der Autoindustrie 1000) Arbeitsstunden pro Beschäftigtem in sechs aufeinander folgenden Wochen verfügbar (zuvor 600 Stunden in vier Wochen) (vgl. genauer Eichhorst/ Marx 2009).
} 
(1) Es ist anzumerken, dass es sich bei den erwähnten Aktionen angesichts der Vielzahl der zeitgleich stattfindenden Entlassungen und Betriebsschließungen um relativ vereinzelte und singuläre Fälle handelte. Diese riefen allerdings ein überproportional starkes Medienecho hervor - u. a. wohl deshalb, weil sie in spektakulärer Weise die Ängste vor sozialen Unruhen symbolisierten und weckten, die zu diesem Zeitpunkt bei Arbeitgebern wie Gesetzgebern (nicht nur in Frankreich) relativ ausgeprägt waren.

(2) Es ging in den stark zugespitzten betrieblichen Kämpfen teilweise um Forderungen, die aus deutscher Perspektive eher moderat erscheinen. Das rechtlich garantierte Minimum von Abfindungszahlungen im Fall einer Betriebsschließung ist in Frankreich relativ niedrig. Standard sind etwa zwei bis drei Monatsgehälter. Die Aushandlung von primes supralegales (Prämien über dem gesetzlichen Niveau) ist daher gängige Praxis im Rahmen von Sozialplanverhandlungen, die allerdings nur dort gelingt, wo ein entsprechender rapport de force vorhanden ist. Dabei ist die Erregung medialen Aufsehens ein gängiges Mittel, um Unternehmensleitungen unter Druck zu setzen nach dem Motto: Je mehr Wirbel es gibt, desto höher sind die Abfindungen. In der Praxis führt dies freilich zu einer starken Differenzierung der gezahlten Prämien, je nachdem, wie viel Angst die entlassenden Unternehmen um ihren Ruf haben, wie finanzstark sie sind, ob sie überhaupt noch weiter existieren und wie effizient und glaubwürdig die Beschäftigtenvertreter in ihren medialen Drohgebärden sind. Zuweilen liegen die auf sehr militantem Wege erkämpften Prämien aus deutscher Sicht daher erstaunlich niedrig. Die New-Fabris-Kollegen etwa mussten letztlich ihre Gasflaschen wieder vom Dach holen gegen das Versprechen von $12.000 €$ Abfindung.

(3) Die oben genannten Aktionen sind also im französischen Kontext längst nicht so spektakulär, wie sie für den deutschen Betrachter wirken. Die sequestration, d.h. das zeitlich befristete Festsetzen von Managern, reiht sich ein in das durchaus gängige Repertoire französischer gewerkschaftlicher Kampfmittel zur Herstellung bzw. Beeinflussung des betrieblichen rapport de force. In besonders zugespitzten Momenten französischer Arbeiterkämpfe wurde es wiederholt eingesetzt (vgl. etwa 1968/69, den Fall Lip-Besancon 1973, oder die Auseinandersetzungen bei Renault-Billancourt 1986). So wird etwa bereits von Jean-Paul Sartre der Spruch kolportiert, wonach es ein emanzipatorischer Schritt sei, „wenn ein Chef seine Beschäftigten um Erlaubnis fragen muss, wenn er pinkeln will.“ Und französische Personalchefs sollen zuweilen prophylaktisch ihre Zahnbürste mit ins Unternehmen bringen, wenn sie einen Sozialplan zu verkünden haben. Obwohl die sequestration häufig als Ausbruch spontaner Wut stilisiert wird, so ist sie doch meist alles andere als das und im Gegenteil in hohem Maße von kodifizierten Regeln geprägt. Als ritualisierte Sprachregelung kann beispielsweise gerade die „Tarnung“ des Geschehens als unkontrollierbare Aktion der Basis gelten. Obwohl sich die Gewerkschaften von den Aktionen gewöhnlich nicht distanzieren, rufen sie weder dazu auf noch bekennen sie sich explizit zu ihnen. Typischerweise wird offiziell betont, dass niemand „entführt“, sondern nur jemand „zur Diskussion zurückgehalten“ wird. Körperliche Gewaltanwendung gegen die „zurückgehaltenen“ Manager ist ein absolutes Tabu. Solange die „Regeln des Spiels“ eingehalten werden, haben die Arbeiterinnen und Arbeiter gewöhnlich nicht mit strafrechtlichen Sanktionen zu rechnen. Dass beispielsweise die Beschäftigten von Continental, die die Präfektur verwüstet hatten, in erster Instanz zu längeren Haftstrafen auf Bewährung verurteilt wurden, war eine Ausnahme. Ihre strafrechtliche Verfolgung sollte signalisieren, dass sie mit ihrer Aktion nicht nur die Grenzen der Legalität, sondern auch jene der Legitimität überschritten hatten. In zweiter Instanz wurden die Haftstrafen dann jedoch zurückgenommen. Insgesamt sprengen also die „Bossnappings“ und Fabrikbesetzungen vom Frühjahr 2009 (denen einige weitere im Herbst folgten) keineswegs das gängige Handlungsrepertoire industrieller Beziehungen in Frankreich. Im Gegenteil: Es sind ritualisierte Formen der Auseinandersetzung, die zum machtpolitischen Spiel gehören und dieses insgesamt eher bestätigen denn verletzen.

(4) Schließlich ist zu betonen, dass die oben erwähnten betrieblichen Kämpfe durchweg defensiven Charakter hatten. Sie sind dem Typus „basisorientierter Protest gegen Betriebsschließungen“"zuzurechnen, der sich dort ereignet, „wo Unternehmen jegliches Interesse an einer Fortsetzung der Produktion - auch bei erheblicher Kostenreduzierung - oder an einem Verkauf des Werks verloren haben. Die Auseinandersetzungen folgen nicht einem vorab definierten strategischen Plan (...), sondern sie entwickeln sich im Kontext dezentraler Willensbildungsprozesse" (Detje et al. 2008, S. 241). Häufig spielt ein moralisches Moment eine große Rolle. Man will sich „nicht wegwerfen lassen wie Dreck“ (Zitat eines Belegschaftsrepräsentanten von New Fabris, zitiert nach Hahn 2009b). Mit einem nachhaltigen Einfluss der Lohnabhängigen auf ihre Ausbeutungsbedingungen haben diese Kämpfe nichts zu tun; sie dokumentieren eher das genaue Gegenteil.

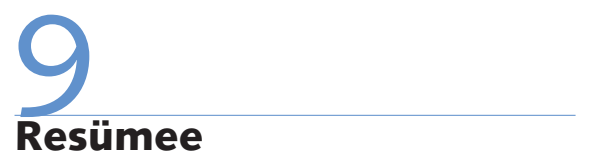

Die Politik der französischen Gewerkschaften in der Wirtschaftskrise muss sowohl nach zeitlichen Phasen als auch nach Akteursebenen differenziert werden: Auf eine begrenzte Mobilisierung von oben und eine ausgeprägte colère collective $\mathrm{zu}$ Beginn des Jahres 2009 folgt die Phase der zersprengten Mobilisierung von unten, d.h. der zugleich vereinzelten wie radikalisierten colère éclatée. Die eher moderate Gewerkschaftspolitik auf der Ebene der Spitzenverbände hat wenig bis nichts zu tun mit den stark zugespitzten, militanten Aktionen auf Betriebsebene im zweiten Halbjahr 2009. Das in Deutschland viel beachtete Phänomen des „Bossnappings“ erscheint in dieser Perspektive eher als relativ ohnmächtige sowie späte Reaktion zersplitterter Akteure nach dem Ende der Hoffnung auf kollektiven Widerstand. Innerhalb der französischen Verhältnisse, wo Konflikte zur Durchsetzung selbst rudimentärer Forderungen oft notwendig sind, haben sie den gängigen Rahmen zugespitzter betrieblicher Konfliktsituationen kaum gesprengt.

Erklärungsbedürftig erscheint im französischen Kontext eher das Zögern der Gewerkschaftszentralen, die offenbar vorhandene Konfliktbereitschaft der Bevölkerung im Frühjahr 2009 für eine entschiedene Mobilisierung gegen die Regierungspolitik zu nutzen. Bei der Erklärung helfen kann sicherlich die Erkenntnis, wonach die Wirtschaftskrise aus Sicht 
der französischen Gewerkschaften „eine Krise in der Krise" darstellt. In dieser Situation existiert ein gewisser Zwang zur Wahrung gewerkschaftlicher Einheit - mit der notwendigen Folge inhaltlicher Mäßigung politischer Strategien. Des Weiteren gewinnt angesichts der eigenen organisatorischen Schwäche die Aufrechterhaltung des dialogue social mit der Regierung an Bedeutung. Die historisch etablierten, impliziten „do ut des“-Beziehungen zwischen Gewerkschaftsdachverbänden und Staat, also das Prinzip des Gebens und Nehmens, sind in Zeiten erodierender Mitgliederzahlen und schwindender Einflussmacht an der betrieblichen Basis für die französischen Gewerkschaften wichtiger denn je.

Fast scheint es daher, als würden die französischen Gewerkschaften in nachholender Entwicklung einen Weg beschreiten, wie er sich seit den 1990er Jahren bei vielen Gewerkschaften Europas als dominante Strategie durchgesetzt hat (vgl. Hassel 2000; Pochet/Fajertag 1997): Die Konstruktion nationaler Solidaritätspakte ist nicht nur eine Reaktion auf die fortschreitende wirtschaftliche Internati- onalisierung und die Durchsetzung nationaler Standortlogiken auch im Bereich der Gewerkschaftspolitik; sie ist auch Ausdruck einer anhaltenden Defensivposition der Gewerkschaften. Angesichts schwindender Mitgliederressourcen und Mobilisierungsfähigkeit bedarf es zunehmend staatlicher Organisationshilfen zur Restituierung gewerkschaftlicher Macht. Dies ist sicherlich ein Erklärungsfaktor (unter anderen), weshalb es nicht nur in Frankreich, sondern tendenziell europaweit während der Wirtschaftskrise kaum zu entschiedenen Ansätzen des Aufbaus gewerkschaftlicher Gegenmacht kam. Stattdessen erlebten die Gewerkschaften in ihrer Rolle als Ordnungsfaktoren eine Renaissance. Verallgemeinerungsfähig am französischen Fallbeispiel ist dabei auch das zunehmende Auseinanderklaffen von Spitzenpolitik und betrieblichen Konflikten, die mangels übergeordneter Koordinierung in zersprengter Weise ausagiert werden und damit letztlich in ihrer politischen Virulenz verpuffen.

Speziell innerhalb der französischen Kultur industrieller Beziehungen stellt sich allerdings die Frage, ob eine gewerkschaft- liche Politik des sozialen Dialogs auf Dauer funktionieren kann. Denn dazu gehören immer (mindestens) zwei. Selbst wenn die konservative Regierung die CGT und die CFDT momentan als Diskussionspartner und als Ordnungsmächte wertschätzt, so hat man die französischen Unternehmer zu einem solchen Verhalten bislang noch immer zwingen müssen. Während eine korporatistische Politik der Organisationssicherung durch politische Bündnisse in Deutschland immerhin seit dem ersten Weltkrieg Tradition hat, birgt eine solche Politik in Frankreich erheblich größere Risiken - und ist daher auf Dauer auch weniger wahrscheinlich. Ob ein WiederUmschwenken in Richtung „Gegenmacht“ bei Bedarf jedoch umstandslos möglich ist, wird sich vielleicht schon bei der anstehenden Diskussion über die Erhöhung des Rentenalters zeigen. Als politische Akteure im Kampf gegen die Wirtschaftskrise haben sich die französischen Gewerkschaften jedenfalls in den Augen vieler Beschäftigter durch ihre zögerliche Politik gehörig desavouiert. Einer Lösung ihrer strukturellen Krisensituation sind sie dadurch keinesfalls näher gekommen.

\section{LITERATUR}

Albert, M. (1991): Capitalisme contre Capitalisme, Paris Amadieu, J.F. (1995): Industrial Relations: Is France a Special Case?, in: British Journal of Industrial Relations 3, S. 345-351

Andolfatto, D./Labbé, D. (2009): Toujours moins! Déclin du syndicalisme à la francaise, Paris

Artus, I. (2008): Interessenhandeln jenseits der Norm. Mittelständische Betriebe und prekäre Dienstleistungsarbeit in Deutschland und Frankreich, Frankfurt a.M./New York

Artus, I. (2010): Mitbestimmung versus Rapport de force: Geschichte und Gegenwart betrieblicher Interessenvertretung im deutsch-französischen Vergleich, Beitrag zum Sammelband "Arbeitswelten und Arbeitsbeziehungen als Gegenstand historischer Forschung - neue Perspektiven auf die Gewerkschaftsgeschichte" ${ }^{\prime}$ Ms. (im Erscheinen)

Beaud, S./Pialoux, M. (2004): Die verlorene Zukunft der Arbeiter. Die Peugeot-Werke von Sochaux-Montbéliard, Konstanz

Berger, S. (Hrsg.) (1981): Organizing Interests in Western Europe. Pluralism, Corporatism and the Transformation of Politics, Cambridge

Béroud, S./Yon, K. (2009): Face à la crise, que fait le movement syndical?, http://www.contretemps.eu/auteurs/sophie-beroud (letzter Zugriff: 20.5.2010)

Dejours, C./Bègue, F. (2009): Suicide et travail: Que faire?, Paris
Detje, R./Menz, W./ Nies, S./Sanné, G./Sauer, D. (2008) : Gewerkschaftliche Kämpfe gegen Betriebsschließungen - Ein Anachronismus?, in: WSI-Mitteilungen 5, S. 238-245

Dribbusch, H. (2008): Streiks in Deutschland, in: WSI (Hrsg.): WSITarifhandbuch 2008, Frankfurt a.M., S. 55-85

Eichhorst, W./Marx, P. (2009): Kurzarbeit: Sinnvoller Konjunkturpuffer oder verlängertes Arbeitslosengeld?, IZA Standpunkte 5, Institut zur Zukunft der Arbeit (IZA), Bonn

Erd, R. (1978): Verrechtlichung industrieller Konflikte. Normative Rahmenbedingungen des dualen Systems der Interessenvertretung, Frankfurt a.M./New York

Gilcher-Holtey, I. (1995): „Die Phantasie an die Macht": Mai 68 in Frankreich, Frankfurt a. M.

Goetschy, J. (1998): France: The limits of Reform, in: Ferner, A./Hyman, R. (Hrsg.): Changing industrial relations in Europe, London, S. 257-394 Hahn, D. (2009a): Eingesperrte „Dikatoren“. Frankreichs Arbeiter wehren sich, in: die tageszeitung (taz) vom 3.4.

Hahn, D. (2009b): „Nicht wegwerfen lassen wie Dreck“. New FabrisBetriebsrat über den Arbeitskampf, in: die tageszeitung (taz) vom 22.7. Hall, P.A./Soskice, D. (Hrsg.) (2001): Varieties of Capitalism. The Institutional Foundations of Comparative Advantage, New York 
Hassel, A. (2000): Bündnisse für Arbeit. Nationale Handlungsfähigkeit im europäischen Regimewettbewerb, in: Politische Vierteljahresschrift 3, S. 498-524

Hassel, A. (2009): Policies and Politics of Social Pacts in Europe, in: European Journal of Industrial Relations 1, S. 7-26

Horn, G.A./Joebges, H./Logeay, C./Sturn, S. (2008): Frankreich: Ein Vorbild für Deutschland? Ein Vergleich wirtschaftspolitischer Strategien mit und ohne Mindestlohn, IMK Report 31, Düsseldorf Institut de Recherches Economiques et Sociales (IRES) (2009):

La France du travail. Donnés, analyses, débats, Paris

Jefferys, S. (2003): Liberté, Égalité and Fraternité at Work. Changing French Employment Relations and Management, Basingstoke/New York Ministère du Travail, des Relations Sociales, de la famille, de la solidarité et de la ville (2008): Représentativité des syndicates. Mode d'emploi, brochure, http://www.travail-solidarite.gouv.fr/IMG/pdf/ Representation_des_syndicats_22_01_09.pdf (letzter Zugriff: 20.5.2010) Moriaux, R. (1998): L'incroyable survie de la CGT, in: Regards sur l'actualité 244, S. 13-23

Müller-Jentsch, W. (1993) (Hrsg.): Konfliktpartnerschaft. Akteure und Institutionen der industriellen Beziehungen, München/Mering
Pernot, J.-M. (2005): Syndicats : lendemains de crise?, Paris Pernot, J.-M. (2010): Introduction, in: Pernot, J.-M.: Syndicats: lendemains de crise?, Paris (im Erscheinen)

Pochet, P./Fajertag, G. (1997): Social Pacts in Europe in the 1990s. Towards a European Social Pact?, in: Pochet, P./Fajertag, G. (Hrsg.): Social Pacts in Europe, Brüssel, S. 9-26

Rehfeldt, U. (2009): La concertation au sommet tourjours d'actualité face à la crise? Theorie du néocorporatisme et analyse compare des relations professionnelles en Europe, in: Chronique internationale de I'IRES 121, S. 40-49

Schulten, T. (2010): Europäischer Tarifbericht des WSI 2009/2010, in: WSI-Mitteilungen 4, S. 196-203

Streeck, W. (1999): Korporatismus in Deutschland: Zwischen Nationalstaat und Europäischer Union, Frankfurt/New York

Traxler, F./Blaschke, S./Kittel, B. (2001): National Labour Relations in Internationalized Markets. A Comparative Study of Institutions, Change and Performance, Oxford

Wildcat (2009): Bossnapping in Frankreich, in: Wildcat 84, www.wildcat-www.de 